\title{
Metodologia para o teste de envelhecimento acelerado em sementes de ervilha
}

\author{
Warley Marcos Nascimento; Raquel A de Freitas; Eliana Marília L Gomes; Aline S Soares \\ Embrapa Hortaliças, C. Postal 218, 70359-970 Brasília-DF; wmn@cnph.embrapa.br; raquel@cnph.embrapa.br
}

\begin{abstract}
RESUMO
A produção de ervilha no Brasil vem utilizando sementes produzidas no próprio país. O presente trabalho teve como objetivo verificar a eficiência do teste de envelhecimento acelerado para avaliar o vigor de sementes de ervilha. Foram utilizados dez lotes de sementes de ervilha, sendo cinco da cultivar Axé (sementes rugosas, indicada para produção de grãos verdes) e cinco da cultivar Mikado (sementes lisas, indicada para produção de grãos secos). Para avaliar a qualidade fisiológica inicial dos lotes foram utilizados os testes de germinação, primeira contagem e emergência de plântulas em campo. Determinou-se também o teor de água das sementes. $\mathrm{O}$ envelhecimento acelerado foi conduzido a $41^{\circ} \mathrm{C}$ por períodos de 24 ; 48 e 72 horas, com e sem uso de solução saturada de $\mathrm{NaCl}$. O experimento foi conduzido no delineamento inteiramente casualizado. $\mathrm{O}$ teste de envelhecimento acelerado mostrou-se eficiente para avaliar o vigor de sementes de ervilha. Quando conduzido sem o uso de solução saturada com $\mathrm{NaCl}$, verificou-se que o período de envelhecimento que permitiu melhor estratificação dos lotes de sementes foi 72 horas. Porém, a porcentagem de germinação após esse período mesmo nos lotes de maior vigor (35\% para a cultivar Axé e 38\% para a cultivar Mikado) foi muito baixa em relação às observadas após apenas 24 horas, superior a $80 \%$ para ambas as cultivares. Quando conduzido com o uso de solução saturada de $\mathrm{NaCl}$, o período de 48 horas, a $41^{\circ} \mathrm{C}$, foi o mais adequado para a classificação dos lotes em diferentes níveis de vigor. Nessas condições, os lotes de maior vigor das cultivares Axé e Mikado apresentaram germinação superior a 68 e 79\%, respectivamente. Os testes de germinação, primeira contagem e emergência de plântulas em campo não apresentaram isoladamente bom poder discriminatório da qualidade fisiológica inicial dos lotes de sementes. Quando seus resultados foram tomados em conjunto, porém, foi possível identificar os lotes de melhor qualidade.
\end{abstract}

Palavras chave: Pisum sativum, vigor, qualidade fisiológica, solução salina, germinação.

\begin{abstract}
\section{Accelerated aging test on pea seeds}

Pea production in Brazil uses seeds produced in the country. The objective of this study was to examine the efficiency of the accelerated aging test for vigor evaluation of pea seeds. Five seed lots of cultivar Axé (wrinkled seeds) and five seed lots of cultivar Mikado (smooth seeds) were used. The initial quality of each seed lot was evaluated by germination test, first counting, and seedling emergence in the field. Seed moisture content was also assessed. The accelerated aging test was set at $41^{\circ} \mathrm{C}$ for periods of $24 ; 48$; and 72 hours, with and without saturated $\mathrm{NaCl}$ solution. The experiment was carried out in a completely randomized design. The accelerated aging test was efficient for vigor evaluation of pea seeds, and the period of 48 hours at $41^{\circ} \mathrm{C}$, using saturated $\mathrm{NaCl}$ solution was the most adequate procedure to indicate vigor levels of pea seeds. However, the seed germination after this period was very low when compared to 24 hour-period ( $80 \%$ ) for both cultivars, even in higher vigor seed lots (35\% for Axé and 38\% for Mikado). In the saturated $\mathrm{NaCl}$ solution, the period of 48 hours at $41^{\circ} \mathrm{C}$ was the most adequate for separate seeds through vigor levels. In these conditions, seed lots of highest vigor showed germination of $68 \%$ and $79 \%$ for Axé and Mikado, respectively. Results of the germination test, first counting, and seedling emergence were not effective in discriminating physiological seed quality when used individually. Nevertheless, when results from these tests were used all together, it was possible to identify the best seed lots.
\end{abstract}

Keywords: Pisum sativum, vigor, physiological quality, saline solution, germination.

\section{(Recebido para publicação em 26 de janeiro de 2006; aceito em 18 de junho de 2007)}

$\mathrm{A}$ ervilha é uma hortaliça de alto valor nutritivo, com amplas alternativas de uso na alimentação. As cultivares utilizadas para produção de ervilha seca possuem sementes redondas e lisas, podendo ser reidratadas para consumo imediato ou enlatadas. Dos grãos secos pode ser obtida ainda a farinha de ervilha, que tem emprego direto na fabricação de sopas instantâneas e na panificação (Pereira, 1989; Giordano, 1997). As cultivares utilizadas para produção de grãos verdes possuem, em geral, sementes rugosas e elevado teor de açúcar (Vieira et al., 2001). Na forma de grãos verdes, a ervilha pode ser consumida in natura, bem como enlatada ou congelada imediatamente após a colheita (Giordano, 1997). A ervilha verde tem sido uma opção de produção para atender às novas demandas do mercado, principalmente de produtos supergelados.

A diferença básica entre as cultivares de sementes lisas e rugosas é sua composição química, principalmente no que se refere aos teores de amido e açúcares. Durante a maturação das sementes, ocorre inicialmente maior acúmulo de açúcares e menor síntese de amido; logo em seguida, o conteúdo de açúcar cai bruscamente e o teor de amido aumenta. Em cultivares de sementes lisas, o primeiro estágio é bem mais curto; sendo que nas sementes rugosas, o acúmulo de açúcar é significante. Com a redução do teor de açúcar durante a maturação, as sementes das cultivares de ervilha-verde apresentam fenótipos caracterizados por enrugamento (sementes rugosas). Estas diferenças de composição química e forma das sementes podem influenciar a qualidade fisiológica e sanitária das mesmas. Por exemplo, as sementes das cultivares de ervilha-verde, por possuírem maior teor de açúcar, são mais suscetíveis ao ataque de fungos (Nascimento \& Cícero, 1991a, b). 
Em se tratando de cultivares de ervilha utilizadas para processamento industrial, onde a uniformidade das épocas e duração do florescimento e maturação são necessárias para obtenção de produto adequado (Mullet \& Wilkinson, 1979), a utilização de testes de vigor, capazes de detectar diferenças significativas de qualidade fisiológica entre lotes de germinação semelhante, torna-se praticamente obrigatória no programa de produção de sementes. Inúmeros estudos têm sido realizados no desenvolvimento, adaptação e padronização dos testes para avaliação do vigor das sementes. Para ervilha, o teste de condutividade elétrica tem apresentado bons resultados, tanto que é recomendado como teste de vigor para a espécie (Hampton \& Tekrony, 1995). No entanto, Marcos Filho (1999b) destaca a importância da utilização de vários testes para avaliação do vigor das sementes, uma vez que estas podem mostrar reações variáveis diante da exposição às diferentes situações específicas de cada teste.

$\mathrm{O}$ envelhecimento acelerado como teste de vigor tem sido um dos mais utilizado, com possibilidade de aplicação para sementes de diversas culturas (McDonald, 1995). Nesse teste, as sementes são submetidas à alta temperatura e umidade relativa elevada por período relativamente curto, sendo, em seguida, colocadas para germinar. Lotes de sementes de alto vigor manterão sua viabilidade quando submetidos a essas condições, enquanto os de baixo vigor terão sua viabilidade reduzida (Association of Official Seed Analysts - AOSA, 1983). O teste de envelhecimento acelerado foi originalmente desenvolvido para determinar o potencial de armazenamento das sementes. No entanto, além de estudos relacionandoo com o potencial de armazenamento das sementes, também têm sido realizados trabalhos para verificar sua eficiência na avaliação do potencial de emergência das plântulas em campo. Em cebola, por exemplo, o envelhecimento acelerado foi um dos testes que mais se associou à emergência de plântulas em campo e à obtenção de mudas vigorosas (Piana et al., 1995).

Embora o teste de envelhecimento acelerado já tenha caminhado suficientemente em direção à padronização, para muitas espécies, ainda há vários estudos em andamento com o objetivo de aprimorar sua metodologia (Marcos Filho, 1999a). A padronização de um teste envolve a identificação de todas as variáveis que influenciam significativamente os resultados e a incorporação de práticas adequadas de controle. No teste de envelhecimento acelerado, as variáveis básicas são temperatura, umidade relativa do ar e período de duração do mesmo. $\mathrm{O}$ teste pode ser conduzido com temperaturas variando entre $41^{\circ} \mathrm{C}$ e $45^{\circ} \mathrm{C}$, sendo que mais recentemente a maioria dos trabalhos indica o uso de $41^{\circ} \mathrm{C}$ (Marcos Filho, 1999a). No entanto, Menezes \& Nascimento (1988) constataram que a temperatura de $37^{\circ} \mathrm{C}$ e o período de 72 horas foram os mais eficientes para avaliação do vigor de sementes de ervilha. Caliari \& Marcos Filho (1990), por sua vez, verificaram que o teste de envelhecimento acelerado conduzido à temperatura de $42^{\circ} \mathrm{C}$ por um período de 48 horas forneceu informações consistentes a respeito do vigor dos lotes de sementes da mesma espécie. Nestes dois estudos, utilizaram apenas cultivares de sementes lisas.

Para a maioria das hortaliças, o teste de envelhecimento acelerado pode apresentar certas limitações, como a desuniformidade de absorção de água entre as amostras, o que pode resultar em deterioração diferenciada, comprometendo os resultados pós-envelhecimento. Visando minimizar esse problema, Jianhua \& McDonald (1996) propuseram, durante a condução do teste, a substituição de água por soluções saturadas de sais. Com esse procedimento, há redução da umidade relativa do ambiente, retardando assim a absorção de água pelas sementes. Isto possibilita ainda redução de infecção por microrganismos durante o teste, o que pode mascarar os resultados.

Assim, o objetivo deste trabalho foi verificar a eficiência do teste de envelhecimento acelerado, com e sem utilização de solução saturada de $\mathrm{NaCl}$, na determinação do potencial fisiológico de sementes lisas e rugosas de ervilha.

\section{MATERIAL E MÉTODOS}

A pesquisa foi conduzida em laboratório da Embrapa Hortaliças em Brasília, DF. Foram utilizadas as culti- vares Axé (sementes rugosas) e Mikado (sementes lisas). Estas são as principais cultivares de ervilha no mercado, destinadas à indústria de grãos verdes e reidratação, respectivamente. Foram utilizados cinco lotes da cultivar Axé e cinco da cultivar Mikado, produzidos nos anos de 2000; 2001; 2003 e 2004, para os quais foram feitas as seguintes determinações:

- Teor de água: foi adotado o método da estufa a $105 \pm 3^{\circ} \mathrm{C}$, durante 24 horas, utilizando-se duas subamostras para cada lote (Brasil, 1992). Os resultados foram expressos em porcentagem na base úmida;

- Germinação: conduzido conforme a metodologia descrita nas Regras para Análise de Sementes (Brasil, 1992);

- Primeira contagem: realizada conjuntamente com o teste de germinação, contabilizando-se o número de plântulas normais presentes no quinto dia após o início do teste.

Já para o envelhecimento acelerado, utilizou-se a metodologia proposta pela AOSA (1983) e descrita por Marcos Filho (1999a). Foram distribuídas 250 sementes sobre tela de alumínio, fixada em caixa plástica de germinação $(11 \mathrm{x}$ $11 \times 3,5 \mathrm{~cm})$, contendo no fundo $40 \mathrm{~mL}$ de água destilada. As caixas contendo as sementes foram fechadas e mantidas a $41^{\circ} \mathrm{C}$, por $24 ; 48$ e 72 horas. Ao término de cada período, as sementes foram submetidas ao teste de germinação, sendo a avaliação da porcentagem de plântulas normais realizada cinco dias após a semeadura. Determinou-se também o teor de água atingido pelas sementes após os diferentes períodos de envelhecimento, visando o monitoramento dos resultados. O teste de envelhecimento acelerado foi também realizado utilizando-se o procedimento proposto por Jianhua \& McDonald (1996), substituindo-se os 40 $\mathrm{mL}$ de água adicionados em cada compartimento individual por igual quantidade de solução saturada de $\mathrm{NaCl}(40 \mathrm{~g}$ de $\mathrm{NaCl} 100 \mathrm{~mL}^{-1}$ de água).

Foi avaliada ainda a emergência das plântulas em campo. As sementes foram semeadas na área experimental da Embrapa Hortaliças e a avaliação foi conduzida com quatro repetições de 50 sementes em linhas de 1,0 m de com- 
primento, espaçadas de $0,2 \mathrm{~m}$ à profundidade média de $3,0 \mathrm{~cm}$. A irrigação foi feita sempre que necessário. A avaliação da porcentagem de emergência das plântulas foi efetuada 15 dias após a semeadura.

O experimento foi conduzido no delineamento inteiramente casualizado, com quatro repetições. Os dados foram submetidos aos testes de normalidade e de homogeneidade de variância, que indicaram a não necessidade de transformação. Em seguida, foram submetidos à análise de variância, sendo a comparação de médias efetuada pelo teste de Tukey, a 5\% de probabilidade.

\section{RESULTADOS E DISCUSSÃO}

Verificou-se que a germinação dos lotes de sementes da cultivar Axé foi semelhante, exceto para o lote 4 (Tabela 1). Para a cultivar Mikado, os lotes 1 e 3 apresentaram germinação estatisticamente superior aos demais, apesar de não diferirem estatisticamente do lote 4 (Tabela 1). O teste de primeira contagem não forneceu boa estratificação dos lotes de sementes da cultivar Axé, porém, observou-se que confirmou os resultados da germinação, indicando o lote 4 como de padrão inferior (Tabela 1). Para a cultivar Mikado, esse teste indicou o lote 5 como sendo de menor vigor (Tabela 1). Embora a primeira contagem do teste de germinação seja considerada um indicativo do vigor, sabese que durante o processo de deterioração das sementes, a redução na velocidade de germinação não está entre os primeiros eventos relacionados no processo de deterioração (Delouche \& Baskin, 1973).

Os resultados obtidos no teste de emergência das plântulas em campo indicaram que as sementes do lote $4 \mathrm{da}$ cultivar Axé apresentaram menor qualidade que as sementes do lote 3 , não diferindo estatisticamente dos demais lotes (Tabela 1). Já para as sementes da cultivar Mikado, os lotes 1 e 3 apresentaram o melhor desempenho (Tabela 1). O teste de emergência de plântulas em campo constitui parâmetro indicador da eficiência dos testes para avaliação do potencial fisiológico dos lotes de sementes (Marcos Filho, 1999b). No entanto,

Tabela 1. Germinação, primeira contagem e emergência de plântulas em campo de sementes de ervilha, cultivares Axé e Mikado. (Germination, first counting and seedling emergence in the field percentage of pea seeds, cultivars Axé and Mikado). Brasília, Embrapa Hortaliças, 2005.

\begin{tabular}{|c|c|c|c|}
\hline Lote & Germinação (\%) & $\begin{array}{c}\text { Primeira contagem } \\
(\%)\end{array}$ & $\begin{array}{c}\text { Emergência de } \\
\text { plântulas em campo } \\
(\%)\end{array}$ \\
\hline \multicolumn{4}{|c|}{ Axé } \\
\hline 1 & $94 a$ & $90 a b$ & $82 a b$ \\
\hline 2 & 94 a & $87 \mathrm{ab}$ & $79 a b$ \\
\hline 3 & 94 a & 93 a & $84 a$ \\
\hline 4 & $82 b$ & $79 \mathrm{~b}$ & $64 \mathrm{~b}$ \\
\hline 5 & $91 a b$ & $85 a b$ & $75 a b$ \\
\hline CV $(\%)$ & 5,58 & 6,39 & 11,37 \\
\hline \multicolumn{4}{|c|}{ Mikado } \\
\hline 1 & 95 a & $90 \mathrm{a}$ & $86 a$ \\
\hline 2 & $85 \mathrm{bc}$ & $83 a$ & $68 \mathrm{~b}$ \\
\hline 3 & $97 \mathrm{a}$ & $90 \mathrm{a}$ & $85 a$ \\
\hline 4 & $91 a b$ & $87 \mathrm{a}$ & $72 b$ \\
\hline 5 & $77 \mathrm{c}$ & $71 \mathrm{~b}$ & $65 \mathrm{~b}$ \\
\hline $\mathrm{CV}(\%)$ & 4,36 & 5,44 & 7,10 \\
\hline
\end{tabular}

Médias seguidas pela mesma letra na coluna não diferem entre si, teste de Tukey, $\mathrm{p} \leq 0,05$ (Means followed by the same letter in the column did not differ from each other, Tukey's test, $\mathrm{p} \leq 0.05)$.

Tabela 2. Teores de água obtidos antes e após os períodos de envelhecimento acelerado tradicional (água) e com solução saturada de $\mathrm{NaCl}, \mathrm{a} 41^{\circ} \mathrm{C}$, em sementes de ervilha, cultivares Axé e Mikado. (Seed moisture content obtained before and after the accelerated aging test with water and $\mathrm{NaCl}$ saturated solution, at $41^{\circ} \mathrm{C}$, in pea seeds, cultivars Axé and Mikado). Brasília, Embrapa Hortaliças, 2005.

\begin{tabular}{|c|c|c|c|c|c|c|c|}
\hline \multirow{2}{*}{ Lote } & \multirow{2}{*}{$\begin{array}{l}\text { Teor de } \\
\text { água } \\
\text { inicial }\end{array}$} & \multicolumn{3}{|c|}{ Tradicional (água) } & \multicolumn{3}{|c|}{ Solução saturada ( $\mathrm{NaCl})$} \\
\hline & & $24 \mathrm{~h}$ & $48 h$ & $72 \mathrm{~h}$ & $24 \mathrm{~h}$ & $48 \mathrm{~h}$ & $72 \mathrm{~h}$ \\
\hline \multicolumn{8}{|c|}{ Axé } \\
\hline 1 & 10,2 & 22,1 & 25,3 & 28,9 & 13,5 & 13,4 & 13,5 \\
\hline 2 & 10,2 & 21,9 & 23,7 & 28,8 & 13,7 & 13,5 & 13,5 \\
\hline 3 & 10,0 & 22,2 & 24,6 & 27,5 & 13,1 & 13,5 & 13,4 \\
\hline 4 & 10,5 & 22,6 & 26,3 & 28,6 & 13,5 & 13,6 & 13,4 \\
\hline 5 & 10,4 & 22,4 & 27,1 & 28,9 & 14,0 & 13,6 & 13,2 \\
\hline \multicolumn{8}{|c|}{ Mikado } \\
\hline 1 & 10,2 & 20,2 & 24,9 & 25,8 & 12,7 & 12,4 & 13,0 \\
\hline 2 & 10,1 & 20,5 & 24,8 & 26,8 & 12,3 & 12,3 & 12,7 \\
\hline 3 & 10,2 & 20,3 & 24,3 & 25,1 & 12,4 & 12,2 & 12,1 \\
\hline 4 & 10,2 & 20,7 & 25,5 & 26,1 & 12,5 & 12,8 & 13,0 \\
\hline 5 & 10,5 & 20,2 & 24,9 & 26,7 & 12,8 & 13,1 & 12,8 \\
\hline
\end{tabular}

o poder de discriminação deste teste não foi decisivo, principalmente para a cultivar Axé, em que somente os lotes 3 e 4 diferiram estatisticamente. Evidenciou-se, assim, a importância da utilização de vários testes para avaliação do vigor das sementes.

As sementes de ervilha envelhecidas pelo método tradicional (com água) apresentaram acréscimos no teor de água com o aumento do período de en- velhecimento. Verificou-se que esse incremento foi mais acentuado no primeiro período de envelhecimento, ou seja, após 24 horas (Tabela 2). A partir deste período, o teor de água das sementes continuou aumentando, porém em proporções inferiores, quando comparadas ao primeiro período de envelhecimento, mostrando, portanto, que as sementes secas, por apresentarem baixo potencial mátrico, absorvem água rapi- 
Tabela 3. Porcentagem de plântulas normais obtida no teste de envelhecimento acelerado tradicional (água) e com solução saturada de $\mathrm{NaCl}$, a $41^{\circ} \mathrm{C}$, em sementes de ervilha, cultivares Axé e Mikado. (Normal seedlings percentage obtained after the accelerated aging test with water and $\mathrm{NaCl}$ saturated solution, at $41^{\circ} \mathrm{C}$, in pea seeds, cultivars Axé and Mikado). Brasília, Embrapa Hortaliças, 2006.

\begin{tabular}{|c|c|c|c|c|c|c|}
\hline \multirow{2}{*}{ Lote } & \multicolumn{3}{|c|}{ Tradicional (água) } & \multicolumn{3}{|c|}{ Solução saturada $(\mathrm{NaCl})$} \\
\hline & $24 \mathrm{~h}$ & $48 \mathrm{~h}$ & $72 \mathrm{~h}$ & $24 \mathrm{~h}$ & $48 h$ & $72 \mathrm{~h}$ \\
\hline \multicolumn{7}{|l|}{ Axé } \\
\hline 1 & 79 a & $43 \mathrm{~b}$ & $21 \mathrm{bc}$ & $88 a$ & 72 a & $71 \mathrm{a}$ \\
\hline 2 & 77 a & 58 a & $17 \mathrm{c}$ & $81 a$ & 74 a & 75 a \\
\hline 3 & $85 a$ & $45 \mathrm{~b}$ & $28 a b$ & $85 a$ & 68 a & 69 a \\
\hline 4 & 79 a & $42 \mathrm{~b}$ & $19 \mathrm{bc}$ & 78 a & $56 \mathrm{~b}$ & $55 \mathrm{~b}$ \\
\hline 5 & $87 \mathrm{a}$ & $51 \mathrm{ab}$ & 35 a & $88 \mathrm{a}$ & $49 \mathrm{~b}$ & $48 \mathrm{~b}$ \\
\hline $\mathrm{CV}(\%)$ & 5,57 & 10,40 & 17,55 & 5,93 & 9,13 & 8,72 \\
\hline \multicolumn{7}{|c|}{ Mikado } \\
\hline$\overline{1}$ & $84 \mathrm{~b}$ & 72 a & $23 \mathrm{~b}$ & $82 a b$ & $79 a b$ & 75 a \\
\hline 2 & $68 \mathrm{c}$ & $52 \mathrm{~b}$ & $21 \mathrm{~b}$ & $74 \mathrm{~b}$ & $62 \mathrm{c}$ & $62 \mathrm{ab}$ \\
\hline 3 & 94 a & 75 a & 38 a & $84 a b$ & 86 a & 79 a \\
\hline 4 & $88 a b$ & 74 a & 32 a & $87 a$ & $74 \mathrm{~b}$ & $67 a$ \\
\hline 5 & $71 \mathrm{c}$ & $40 \mathrm{~b}$ & $10 \mathrm{c}$ & $74 \mathrm{~b}$ & $58 \mathrm{c}$ & $43 \mathrm{~b}$ \\
\hline CV (\%) & 4,92 & 9,39 & 12,74 & 6,95 & 7,52 & 15,95 \\
\hline
\end{tabular}

Médias seguidas pela mesma letra na coluna não diferem entre si, teste de Tukey, $p \leq 0,05$ (Means followed by the same letter in the column did not differ from each other, Tukey's test, $\mathrm{p} \leq 0.05)$.

damente quando colocadas em atmosfera úmida (Bewley \& Black, 1994). Verificou-se ainda que com a utilização de solução saturada de $\mathrm{NaCl}$ obteve-se valores de teores de água inferiores aos observados para as sementes envelhecidas pelo método tradicional (Tabela 2).

$\mathrm{O}$ envelhecimento acelerado pelo método tradicional, por um período de 24 horas, não separou os lotes de sementes da cultivar Axé em níveis de vigor, embora o mesmo período de envelhecimento tenha sido suficiente para indicar as sementes dos lotes 2 e 5 da cultivar Mikado como as de menor vigor (Tabela 3). Quando o período de envelhecimento, ainda pelo método tradicional, foi estendido para 48 horas, passouse a observar diferenças de vigor entre os lotes de sementes da cultivar Axé. Nesta situação os lotes 2 e 5 da cultivar Axé foram os de melhor desempenho (Tabela 3). Já para a cultivar Mikado, após 48 horas, observou-se maior vigor das sementes nos lotes 1; 3 e 4. Empregando esse mesmo método e período de envelhecimento, porém sob temperatura de $42^{\circ} \mathrm{C}$, na avaliação do potencial fisiológico de sementes de ervilha, Caliari \& Marcos Filho (1990) verificaram que o teste de envelhecimento acelerado forneceu informações consistentes quanto ao vigor dos lotes. Contudo, com o decorrer do armazenamento, a separação dos lotes não se mostrou eficiente, sugerindo certa severidade das condições do teste.

Quando o período de envelhecimento passou a ser de 72 horas, conduzido com água, as sementes dos lotes 5 e 3 da cultivar Axé foram as de desempenho superior (Tabela 3). Ainda no período de envelhecimento de 72 horas, conduzido pelo método tradicional, confirmou-se para a cultivar Mikado, os lotes 3 e 4 como os de melhor vigor e, o lote 5, como o de menor vigor. Constata-se que o teste de envelhecimento acelerado, conduzido de forma tradicional, a $41^{\circ} \mathrm{C}$ por 72 horas, permitiu estratificar os lotes de sementes das duas cultivares estudadas em maior número de níveis de vigor. No entanto, verificou-se que o estresse a que as sementes foram submetidas reduziu acentuadamente a germinação obtida nesse procedimento. Além disso, observou-se aumento no coeficiente de variação à medida que aumentou o período de exposição ao envelhecimento. Situação semelhante foi observada por Nascimento et al. (1993), em sementes de tomate.
Para a cultivar Axé, o envelhecimento acelerado com solução saturada, conduzido a $41^{\circ} \mathrm{C}$ durante 24 horas, à semelhança do que foi observado no método tradicional, nesse mesmo período, não permitiu separação dos lotes em níveis de vigor das sementes (Tabela 3). Esse procedimento tampouco forneceu uma clara estratificação dos lotes da cultivar Mikado em função dos níveis de vigor das sementes. O período de 48 horas com solução saturada permitiu entretanto classificar os lotes da cultivar Axé em dois grupos em função do vigor das sementes: lotes com alto vigor (1; 2 e 3$)$ e lotes com baixo vigor (4 e 5). Esse período de exposição ao envelhecimento também proporcionou uma boa estratificação dos lotes de sementes da cultivar Mikado.

No período de 72 horas, conduzido com solução saturada, a classificação dos lotes da cultivar Axé em função do vigor das sementes foi idêntica à obtida para o período de exposição por 48 horas, nesse mesmo método de envelhecimento. Desta forma, o período de 48 horas é mais indicado, uma vez que o menor período de execução é uma característica desejável em um teste de vigor e, nesse caso, possibilita a economia de energia elétrica (Ramos et al., 2004). O envelhecimento por 48 horas, com solução saturada, classificou os lotes da cultivar Mikado de forma semelhante à indicada pelo teste de germinação (Tabela 1). No entanto, Menezes \& Nascimento (1988) constataram que a temperatura de $37^{\circ} \mathrm{C}$ e o período de 72 horas foram os mais eficientes para avaliação do vigor de sementes de ervilha.

Observou-se que o teste de envelhecimento acelerado modificado reduz a absorção de água das sementes (Tabela 2). Assim, a utilização de solução saturada proporcionou efeitos menos drásticos do que os provocados pelo envelhecimento tradicional no que se refere ao processo de deterioração das sementes (Tabela 3). Resultados semelhantes foram obtidos por Torres (2005a) em sementes de pepino, e segundo o autor, a utilização de solução saturada possibilitou a obtenção de resultados menos drásticos e mais uniformes, sem afetar a eficiência do teste de envelhecimento acelerado.

Os resultados obtidos no presente trabalho indicaram o teste de envelhe- 
cimento acelerado modificado (com solução saturada), conduzido a $41^{\circ} \mathrm{C}$ por 48 horas, como o método mais adequado para classificação dos lotes de sementes de ervilha em níveis de vigor. Maior eficiência do teste de envelhecimento acelerado com uso de solução saturada de sal na classificação de sementes com diferentes níveis de vigor também foi observado em sementes de pimentão (Panobianco \& Marcos Filho, 1998), cenoura (Rodo et al., 2000), melão (Torres \& Marcos Filho, 2003), pepino (Torres, 2005a) e pimenta-malagueta (Torres, 2005b).

\section{REFERÊNCIAS}

ASSOCIATION OF OFFICIAL SEED ANALYSTS. 1983. Seed vigor testing handbook. East lansing: AOSA. 88p. (Contribution 32).

BEWLEY JD; BLACK, M. 1994. Seeds: physiology of development and germination. 2. ed. New York: Plenum Press. 445p.

BRASIL. 1992. Ministério da Agricultura e Reforma Agrária. Regras para análise de sementes. Brasília: SNDA/DNDV/CLAV. 365p.

CALIARI MF; MARCOS FILHO J. 1990. Comparação entre métodos para avaliação da qualidade fisiológica de sementes de ervilha (Pisum sativum L.). Revista Brasileira de Sementes 12: 52-73.

DELOUCHE JC; BASKIN CC. 1973. Accelerated aging techniques for predicting the relative storability of seed lots. Seed Science and Technology 1: 427-452.
GIORDANO LB. 1997. Cultivo da ervilha. Brasília: EMBRAPA-CNPH. 19p. (Instruções Técnicas 1).

HAMPTON JG; TEKRONY DM. 1995. Handbook of vigour test methods. Zurich: The International Seed Testing Association. $3^{\text {rd }}$ ed. 117p.

JIANHUA Z; Mc DONALD MB. 1996. The saturated salt accelerated aging test for smallseeded crops. Seed Science and Technology 25: 123-131.

MARCOS FILHO J. 1999a. Teste de envelhecimento acelerado. In: KRZYZANOWSKI FC; VIEIRA RD; FRANÇA NETO JB (eds). Vigor de sementes: conceitos e testes. Londrina: ABRATES. cap.3., p.1-24.

MARCOS FILHO J. 1999b. Testes de vigor: importância e utilização. In: KRZYZANOWSKI FC; VIEIRA RD; FRANÇA NETO JB (eds). Vigor de sementes: conceitos e testes. Londrina: ABRATES. p.1-21.

McDONALD MB. 1995. Standardization of seed vigour tests. In: CONGRESS OF THE INTERNATIONAL SEED TESTING ASSOCIATION, 24. Proceedings... Copenhagen: ISTA. p.88-97.

MENEZES JE; NASCIMENTO WM. 1988. Teste de envelhecimento precoce em sementes de ervilha (Pisium sativum L.). Horticultura Brasileira 6: 63 (suplemento).

MULLET JH; WILKINSON RJ. 1979. The relationship between amounts of electrolyte lost on leaching seed of Pisum sativum and some parameters of plant growth. Seed Science and Technology 7: 393-398.

NASCIMENTO WM; BARROS BCG; PESSOA HBSV. 1993. Teste de envelhecimento acelerado em sementes de tomate. Revista Brasileira de Sementes 15: 251-253.

NASCIMENTO WM; CÍCERO SM. 1991a. Qualidade de sementes de ervilha tratada com fungicida. I: qualidade sanitária. Revista Brasileira de Sementes 13: 5-12.
NASCIMENTO WM; CÍCERO SM. 1991b. Qualidade de sementes de ervilha tratadas com fungicida. II: qualidade fisiológica. Revista Brasileira de Sementes 13: 13-19.

PANOBIANCO M; MARCOS FILHO J. 1998. Comparação entre métodos para avaliação da qualidade fisiológica de semente de pimentão. Revista Brasileira de Sementes 20: 306-310.

PEREIRA AS. 1989. Ervilha: uma fonte de nutrientes. Informe Agropecuário 14: 52-54.

PIANAZ; TILLMANN MAA; MINAMI K. 1995. Avaliação da qualidade fisiológica de sementes de cebola e sua relação com a produção de mudas vigorosas. Revista Brasileira de Sementes 17: 149-153.

RAMOS NP; FLOR EPO; MENDONÇA EAF; MINAMI K. 2004. Envelhecimento acelerado em sementes de rúcula (Eruca sativa L.). Revista Brasileira de Sementes 26: 98-103.

RODO AB; PANOBIANCO M; MARCOS FILHO J. 2000. Metodologia alternativa do teste de envelhecimento acelerado para sementes de cenoura. Scientia Agricola 57: 289-292.

TORRES SB. 2005a. Envelhecimento acelerado em sementes de pepino com e sem solução salina saturada. Horticultura Brasileira 23. 303-306.

TORRES SB. 2005b. Envelhecimento acelerado em sementes de pimenta-malagueta (Capsicum frutescens L.). Revista Ciência Agronômica 36: 98-104.

TORRES SB; MARCOS FILHO J. 2003. Accelerated aging of melon seeds. Scientia Agricola 60: 77-82.

VIEIRA RF, VIEIRA C, VIEIRA RF. 2001. Leguminosas graníferas. Viçosa: UFV. 206p. 